\begin{tabular}{|c|c|c|c|c|c|c|c|c|c|c|c|}
\hline \multirow{2}{*}{ Participants } & \multirow{2}{*}{ miRNA pairs } & \multicolumn{5}{|c|}{ CNTR } & \multicolumn{5}{|c|}{ RTT } \\
\hline & & Sens & Spec & Accur & AUC & P-value & Sens & Spec & Accur & AUC & $P$-value \\
\hline \multirow{13}{*}{ 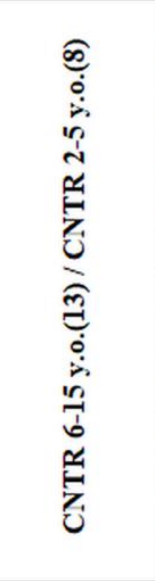 } & $\mathrm{miR}-146 \mathrm{a} / \mathrm{miR}-125 \mathrm{~b}$ & 0.89 & 0.85 & 0.86 & 0.9 & $3.10 \mathrm{E}-03$ & 0.8 & 0.45 & 0.56 & 0.52 & $2.80 \mathrm{E}-01$ \\
\hline & miR-432-5p / miR-125b & 0.89 & 0.77 & 0.82 & 0.86 & $8.10 \mathrm{E}-03$ & 0.57 & 0.6 & 0.59 & 0.68 & $2.50 \mathrm{E}-01$ \\
\hline & miR-107 / miR-125b & 0.8 & 0.79 & 0.8 & 0.89 & $3.80 \mathrm{E}-03$ & 0.67 & 0.3 & 0.42 & 0.54 & $1.50 \mathrm{E}-01$ \\
\hline & miR-335-5p / miR-125b & 0.9 & 0.7 & 0.78 & 0.88 & $5.60 \mathrm{E}-03$ & 0.8 & 0.45 & 0.56 & 0.68 & $2.50 \mathrm{E}-01$ \\
\hline & $\mathrm{miR}-411-5 p / \mathrm{miR}-323-3 p$ & 0.78 & 0.77 & 0.77 & 0.9 & $1.60 \mathrm{E}-03$ & 0.26 & 0.48 & 0.41 & 0.46 & $2.10 \mathrm{E}-01$ \\
\hline & $\mathrm{miR}-206 / \mathrm{miR}-125 \mathrm{~b}$ & 0.89 & 0.69 & 0.77 & 0.87 & $5.60 \mathrm{E}-03$ & 0.8 & 0.73 & 0.75 & 0.81 & $8.60 \mathrm{E}-02$ \\
\hline & miR-433-3p / miR-125b & 0.69 & 0.8 & 0.76 & 0.87 & $9.70 \mathrm{E}-03$ & 0.58 & 0.62 & 0.61 & 0.74 & $1.30 \mathrm{E}-01$ \\
\hline & $\mathrm{miR}-134$ / miR-125b & 0.69 & 0.8 & 0.76 & 0.88 & $5.60 \mathrm{E}-03$ & 0.64 & 0.48 & 0.53 & 0.71 & $2.10 \mathrm{E}-01$ \\
\hline & $\mathrm{miR}-155$ / miR-125b & 0.82 & 0.73 & 0.76 & 0.89 & $4.60 \mathrm{E}-03$ & 0.56 & 0.34 & 0.41 & 0.55 & $4.10 \mathrm{E}-01$ \\
\hline & miR-4ll-5p / miR-125b & 0.69 & 0.79 & 0.75 & 0.86 & $5.60 \mathrm{E}-03$ & 0.8 & 0.55 & 0.62 & 0.74 & $1.50 \mathrm{E}-01$ \\
\hline & miR-107 / miR-132-3p & 0.69 & 0.79 & 0.75 & 0.87 & $4.60 \mathrm{E}-03$ & 0.47 & 0.32 & 0.37 & 0.46 & $1.80 \mathrm{E}-01$ \\
\hline & miR-29b-5p / miR-125b & 0.78 & 0.69 & 0.73 & 0.87 & $9.70 \mathrm{E}-03$ & 0.6 & 0.27 & 0.38 & 0.52 & $2.80 \mathrm{E}-01$ \\
\hline & miR-146a / miR-132-3p & 0.75 & 0.67 & 0.7 & 0.86 & $5.60 \mathrm{E}-03$ & 0.51 & 0.47 & 0.48 & 0.59 & $4.10 \mathrm{E}-01$ \\
\hline \multirow{10}{*}{ 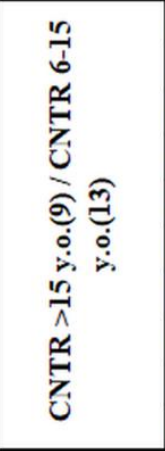 } & miR-335-5p / miR-134 & 0.92 & 0.88 & 0.9 & 0.99 & 1.20E-04 & 0.45 & 0.56 & 0.5 & 0.61 & $4.10 \mathrm{E}-01$ \\
\hline & miR-146a / miR-134 & 0.83 & 0.74 & 0.79 & 0.91 & $1.70 \mathrm{E}-03$ & 0.58 & 0.36 & 0.48 & 0.58 & $4.70 \mathrm{E}-01$ \\
\hline & miR-491-5p / miR-134 & 0.8 & 0.78 & 0.79 & 0.94 & $6.20 \mathrm{E}-04$ & 0.49 & 0.48 & 0.48 & 0.56 & 4.70E-01 \\
\hline & miR-107 / miR-134 & 0.85 & 0.63 & 0.76 & 0.94 & $1.00 \mathrm{E}-03$ & 0.64 & 0.44 & 0.55 & 0.66 & $3.20 \mathrm{E}-01$ \\
\hline & miR-107 / miR-409-3p & 0.79 & 0.7 & 0.76 & 0.9 & $3.30 \mathrm{E}-03$ & 0.53 & 0.64 & 0.58 & 0.68 & $2.50 \mathrm{E}-01$ \\
\hline & miR-18la-5p / miR-134 & 0.85 & 0.63 & 0.76 & 0.91 & $1.70 \mathrm{E}-03$ & 0.6 & 0.63 & 0.62 & 0.71 & $2.50 \mathrm{E}-01$ \\
\hline & let-7b / miR-125b & 0.81 & 0.66 & 0.75 & 0.87 & $7.50 \mathrm{E}-03$ & 0.58 & 0.35 & 0.47 & 0.54 & $3.80 \mathrm{E}-01$ \\
\hline & miR-335-5p / miR-409-3p & 0.83 & 0.54 & 0.72 & 0.93 & $3.30 \mathrm{E}-03$ & 0.39 & 0.71 & 0.54 & 0.62 & $3.80 \mathrm{E}-01$ \\
\hline & miR-16 / miR-125b & 0.65 & 0.83 & 0.72 & 0.87 & $7.50 \mathrm{E}-03$ & 0.33 & 0.6 & 0.45 & 0.52 & $2.20 \mathrm{E}-01$ \\
\hline & miR-335-5p / miR-323-3p & 0.68 & 0.74 & 0.7 & 0.86 & $9.20 \mathrm{E}-03$ & 0.43 & 0.64 & 0.53 & 0.63 & $3.00 \mathrm{E}-01$ \\
\hline
\end{tabular}

\title{
S2a Table
}




\begin{tabular}{|c|c|c|c|c|c|c|c|c|c|c|c|}
\hline \multirow{2}{*}{ Participants } & \multirow{2}{*}{ miRNA pairs } & \multicolumn{5}{|c|}{ RTT } & \multicolumn{5}{|c|}{ CNTR } \\
\hline & & Sens & Spec & Accur & AUC & P-value & Sens & Spec & Accur & AUC & P-value \\
\hline \multirow{23}{*}{ 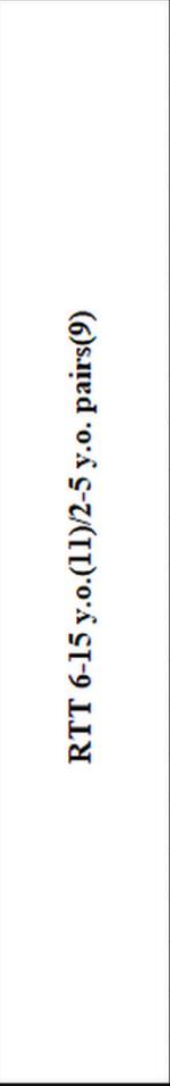 } & miR-122 / miR-155 & 0.91 & 0.89 & 0.9 & 0.95 & $9.10 \mathrm{E}-04$ & 0.38 & 0.61 & 0.46 & 0.55 & $4.30 \mathrm{E}-01$ \\
\hline & miR-122 / miR-335-5p & 0.91 & 0.89 & 0.9 & 0.94 & $1.50 \mathrm{E}-03$ & 0.36 & 0.46 & 0.4 & 0.45 & $1.60 \mathrm{E}-01$ \\
\hline & miR-132-3p / miR-155 & 0.91 & 0.89 & 0.9 & 0.96 & $5.40 \mathrm{E}-04$ & 0.43 & 0.59 & 0.49 & 0.55 & $3.70 \mathrm{E}-01$ \\
\hline & miR-122 / miR-146a & 0.82 & 0.89 & 0.85 & 0.92 & $3.10 \mathrm{E}-03$ & 0.36 & 0.58 & 0.44 & 0.5 & $2.50 \mathrm{E}-01$ \\
\hline & miR-122 / miR-491-5p & 0.82 & 0.89 & 0.85 & 0.92 & $3.10 \mathrm{E}-03$ & 0.32 & 0.64 & 0.44 & 0.51 & $2.90 \mathrm{E}-01$ \\
\hline & miR-122 / miR-16 & 0.84 & 0.8 & 0.83 & 0.89 & $9.20 \mathrm{E}-03$ & 0.46 & 0.38 & 0.43 & 0.55 & $3.70 \mathrm{E}-01$ \\
\hline & $\mathrm{miR}-122$ / miR-29b-5p & 0.73 & 0.89 & 0.8 & 0.93 & $2.50 \mathrm{E}-03$ & 0.31 & 0.63 & 0.43 & 0.49 & $2.20 \mathrm{E}-01$ \\
\hline & miR-122 / miR-107 & 0.73 & 0.89 & 0.8 & 0.92 & $3.90 \mathrm{E}-03$ & 0.31 & 0.63 & 0.43 & 0.49 & $2.50 \mathrm{E}-01$ \\
\hline & $\mathrm{miR}-132-3 \mathrm{p} / \mathrm{miR}-323-3 \mathrm{p}$ & 0.82 & 0.78 & 0.8 & 0.89 & $7.50 \mathrm{E}-03$ & 0.54 & 0.38 & 0.48 & 0.63 & $4.30 \mathrm{E}-01$ \\
\hline & miR-122 / miR-432-5p & 0.76 & 0.82 & 0.79 & 0.92 & $1.90 \mathrm{E}-03$ & 0.44 & 0.48 & 0.45 & 0.54 & $4.30 \mathrm{E}-01$ \\
\hline & miR-122 / miR-411-5p & 0.76 & 0.82 & 0.79 & 0.92 & $2.50 \mathrm{E}-03$ & 0.42 & 0.54 & 0.47 & 0.56 & $4.60 \mathrm{E}-01$ \\
\hline & $\mathrm{miR}-132-3 p / \mathrm{miR}-432-5 p$ & 0.85 & 0.69 & 0.78 & 0.89 & $7.50 \mathrm{E}-03$ & 0.44 & 0.48 & 0.45 & 0.56 & $4.00 \mathrm{E}-01$ \\
\hline & miR-122 / miR-409-3p & 0.73 & 0.78 & 0.75 & 0.88 & $6.10 \mathrm{E}-03$ & 0.49 & 0.66 & 0.56 & 0.68 & $2.70 \mathrm{E}-01$ \\
\hline & miR-18la-5p / miR-411-5p & 0.73 & 0.78 & 0.75 & 0.86 & $2.00 \mathrm{E}-02$ & 0.46 & 0.5 & 0.48 & 0.6 & $4.30 \mathrm{E}-01$ \\
\hline & let-7b/ miR-155 & 0.79 & 0.64 & 0.73 & 0.86 & $7.50 \mathrm{E}-03$ & 0.56 & 0.52 & 0.55 & 0.69 & $2.00 \mathrm{E}-01$ \\
\hline & miR-132-3p/miR-411-5p & 0.67 & 0.81 & 0.73 & 0.86 & $1.10 \mathrm{E}-02$ & 0.43 & 0.46 & 0.44 & 0.54 & $4.30 \mathrm{E}-01$ \\
\hline & $\mathrm{miR}-323-3 p / \mathrm{miR}-432-5 p$ & 0.7 & 0.75 & 0.72 & 0.86 & $7.50 \mathrm{E}-03$ & 0.33 & 0.54 & 0.41 & 0.48 & $2.50 \mathrm{E}-01$ \\
\hline & let-7b / miR-432-5p & 0.73 & 0.67 & 0.7 & 0.86 & $1.10 \mathrm{E}-02$ & 0.57 & 0.46 & 0.53 & 0.6 & $4.30 \mathrm{E}-01$ \\
\hline & miR-122 / miR-323-3p & 0.58 & 0.83 & 0.69 & 0.9 & $3.90 \mathrm{E}-03$ & 0.45 & 0.48 & 0.46 & 0.6 & $4.30 \mathrm{E}-01$ \\
\hline & miR-122 / miR-433-3p & 0.57 & 0.81 & 0.68 & 0.89 & $3.90 \mathrm{E}-03$ & 0.43 & 0.46 & 0.44 & 0.56 & $4.60 \mathrm{E}-01$ \\
\hline & miR-409-3p/miR-432-5p & 0.52 & 0.85 & 0.67 & 0.85 & $1.10 \mathrm{E}-02$ & 0.23 & 0.63 & 0.38 & 0.41 & $1.00 \mathrm{E}-01$ \\
\hline & miR-18la-5p / miR-155 & 0.59 & 0.72 & 0.65 & 0.85 & $2.00 \mathrm{E}-02$ & 0.62 & 0.63 & 0.62 & 0.66 & $2.70 \mathrm{E}-01$ \\
\hline & miR-122 / miR-181a-5p & 0.49 & 0.8 & 0.63 & 0.86 & $1.40 \mathrm{E}-02$ & 0.38 & 0.5 & 0.43 & 0.52 & $2.50 \mathrm{E}-01$ \\
\hline \multirow{20}{*}{ 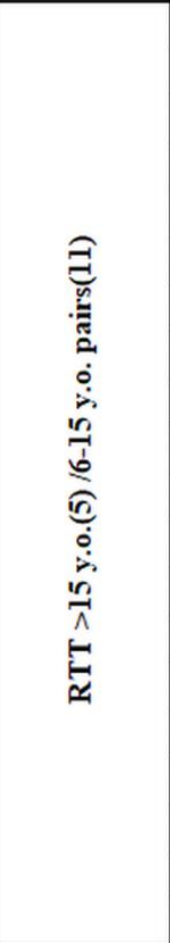 } & miR-122 / miR-491-5p & 0.8 & 0.91 & 0.87 & 0.94 & $6.10 \mathrm{E}-03$ & 0.48 & 0.41 & 0.44 & 0.41 & $1.10 \mathrm{E}-01$ \\
\hline & miR-122 / miR-181a-5p & 0.8 & 0.91 & 0.87 & 0.93 & $8.40 \mathrm{E}-03$ & 0.46 & 0.4 & 0.43 & 0.5 & $1.90 \mathrm{E}-01$ \\
\hline & miR-122 / miR-29b-5p & 0.83 & 0.85 & 0.84 & 0.93 & $8.40 \mathrm{E}-03$ & 0.32 & 0.58 & 0.47 & 0.43 & 1.40E-01 \\
\hline & miR-335-5p / miR-49l-5p & 0.83 & 0.85 & 0.84 & 0.95 & $3.10 \mathrm{E}-03$ & 0.44 & 0.46 & 0.45 & 0.56 & $4.20 \mathrm{E}-01$ \\
\hline & $\mathrm{miR}-122$ / miR-155 & 0.82 & 0.84 & 0.83 & 0.91 & $1.50 \mathrm{E}-02$ & 0.34 & 0.55 & 0.46 & 0.48 & $2.50 \mathrm{E}-01$ \\
\hline & miR-335-5p / miR-18la-5p & 0.81 & 0.83 & 0.82 & 0.89 & $2.60 \mathrm{E}-02$ & 0.53 & 0.52 & 0.52 & 0.63 & $2.70 \mathrm{E}-01$ \\
\hline & miR-411-5p/miR-491-5p & 0.8 & 0.82 & 0.81 & 0.88 & $2.60 \mathrm{E}-02$ & 0.74 & 0.44 & 0.56 & 0.73 & $1.30 \mathrm{E}-01$ \\
\hline & miR-122 / miR-107 & 0.85 & 0.77 & 0.8 & 0.92 & $1.10 \mathrm{E}-02$ & 0.33 & 0.46 & 0.41 & 0.41 & $1.00 \mathrm{E}-01$ \\
\hline & miR-433-3p / miR-491-5p & 0.85 & 0.77 & 0.8 & 0.94 & $4.40 \mathrm{E}-03$ & 0.51 & 0.42 & 0.45 & 0.53 & $3.70 \mathrm{E}-01$ \\
\hline & miR-122 / miR-146a & 0.63 & 0.85 & 0.78 & 0.89 & $1.50 \mathrm{E}-02$ & 0.32 & 0.52 & 0.44 & 0.45 & $1.40 \mathrm{E}-01$ \\
\hline & $\mathrm{miR}-155$ / miR-491-5p & 0.82 & 0.75 & 0.77 & 0.88 & $2.00 \mathrm{E}-02$ & 0.29 & 0.4 & 0.35 & 0.45 & $4.70 \mathrm{E}-02$ \\
\hline & $\mathrm{miR}-433-3 p / \mathrm{miR}-181 \mathrm{a}-5 \mathrm{p}$ & 0.8 & 0.73 & 0.75 & 0.87 & $2.00 \mathrm{E}-02$ & 0.73 & 0.58 & 0.64 & 0.74 & 1.30E-01 \\
\hline & miR-432-5p/miR-491-5p & 0.8 & 0.73 & 0.75 & 0.87 & $2.60 \mathrm{E}-02$ & 0.7 & 0.42 & 0.53 & 0.65 & $2.70 \mathrm{E}-01$ \\
\hline & miR-335-5p / miR-146a & 0.8 & 0.73 & 0.75 & 0.88 & $2.00 \mathrm{E}-02$ & 0.56 & 0.62 & 0.59 & 0.75 & $1.40 \mathrm{E}-01$ \\
\hline & let-7b / miR-49l-5p & 0.6 & 0.82 & 0.75 & 0.86 & $2.60 \mathrm{E}-02$ & 0.33 & 0.38 & 0.36 & 0.39 & $5.40 \mathrm{E}-02$ \\
\hline & miR-335-5p / miR-107 & 0.86 & 0.69 & 0.74 & 0.94 & $6.10 \mathrm{E}-03$ & 0.49 & 0.34 & 0.4 & 0.5 & $2.50 \mathrm{E}-01$ \\
\hline & $\mathrm{miR}-122$ / miR-132-3p & 0.42 & 0.86 & 0.72 & 0.88 & $1.50 \mathrm{E}-02$ & 0.46 & 0.47 & 0.47 & 0.54 & $3.40 \mathrm{E}-01$ \\
\hline & miR-146a / miR-491-5p & 0.58 & 0.78 & 0.72 & 0.87 & $1.50 \mathrm{E}-02$ & 0.36 & 0.5 & 0.44 & 0.51 & $3.40 \mathrm{E}-01$ \\
\hline & miR-16 / miR-491-5p & 0.85 & 0.58 & 0.67 & 0.85 & $3.40 \mathrm{E}-02$ & 0.33 & 0.38 & 0.36 & 0.37 & $4.10 \mathrm{E}-02$ \\
\hline & miR-433-3p/miR-107 & 0.67 & 0.61 & 0.63 & 0.86 & $4.40 \mathrm{E}-02$ & 0.55 & 0.45 & 0.49 & 0.57 & $4.70 \mathrm{E}-01$ \\
\hline
\end{tabular}

S2b Table 